\section{Should we ignore western blots when selecting antibodies for other applications?}

To the Editor: We read the commentary "A proposal for validation of antibodies" by Uhlen et al. with interest ${ }^{1}$. The authors explain that "Because of differences in protein conformation and target accessibility, antibodies that perform well in one context may perform inadequately in others", and they conclude that "approaches for antibody validation must be carried out in an application- and context-specific manner".

Assessing antibody specificity in immunohistochemistry (IHC) and immunofluorescence microscopy (IF) is difficult since there is no comprehensive and definitive source of information about the distribution of proteins in tissues and subcellular compartments. It is therefore common to use results from western blotting (WB) analyses as supportive evidence. In fact, most of the antibodies used in IF and IHC were initially selected on the basis of their performance in WB analyses, as manufacturers often use WB as a first-pass test in product development. The expectation is that antibodies that stain a single band corresponding to the mass of the intended target (i.e. confirmatory) are more likely to be specific in IF and IHC analyses than those that are evidently cross-reactive in WB analyses (i.e. nonconfirmatory).

Strict adherence to the principle of application-specific validation would imply that WB results have no relevance for IF and IHC analyses and that researchers should disregard non-confirmatory WBs when selecting antibodies for use in these applications. This seems counterintuitive, and there is evidence that WB can uncover cross-reactivity and trigger a more cautious interpretation of subsequent IF and IHC data ${ }^{2-6}$.

We would like to ask the authors to clarify their opinion about the predictive value of confirmatory versus non-confirmatory $\mathrm{WB}$ analyses for antibody specificity in IF and IHC. Is there compelling evidence that cross-reactivity in WB has no relevance?

\section{ACKNOWLEDGMENTS}

F.L.-J. receives funding from the $\mathrm{K} G$ Jebsen Foundation.

\section{AUTHOR CONTRIBUTIONS}

F.L.-J. and M.D.B. selected the references and wrote the text.

\section{COMPETING FINANCIAL INTERESTS}

The authors declare competing financial interests: details are available in the online version of the paper.

\section{Fridtjof Lund-Johansen ${ }^{1,2}$ \& Michael D Browning ${ }^{3}$}

${ }^{1} \mathrm{~K}$ G Jebsen Center for Cancer Immunotherapy, Oslo University Hospital, Oslo, Norway. ${ }^{2}$ Department of Immunology, Oslo University Hospital, Oslo, Norway. ${ }^{3}$ PhosphoSolutions, Colorado Bioscience Park, Aurora, Colorado, USA. e-mail: fridtjol@gmail.com
1. Uhlen, M. et al. Nat. Methods 13, 823-827 (2016)

2. Lukinavičius, G., Lavogina, D., Gönczy, P. \& Johnsson, K. Biotechniques 55, 111-114 (2013).

3. Elliott, S. et al. Blood 107, 1892-1895 (2006).

4. Jensen, B.C., Swigart, P.M. \& Simpson, P.C. Naunyn Schmiedebergs Arch. Pharmacol. 379, 409-412 (2009).

5. Lobert, V.H. et al. EMBO Rep. 14, 10-11 (2013).

6. Schuster, C. et al. Histopathology 60 6B, E37-E50 (2012).

Uhlen replies: In the report ${ }^{1}$ from the International Working Group for Antibody Validation (IWGAV), we concluded that "approaches for antibody validation must be carried out in an application- and context-specific manner." Our argument is that samples are treated differently in different applications and that this influences the epitopes exposed on the target protein, which might have profound consequences for the ability of a given antibody to bind specifically to its target. As an example, proteins that are analyzed by immunohistochemistry (IHC) are normally first cross-linked with formalin and then heated to very high temperatures (normally $>100^{\circ} \mathrm{C}$ ) in a procedure that is sometimes termed 'epitope retrieval'. Obviously, this procedure might influence the target protein differently than the procedure used to prepare proteins for a western blot, in which the sample is instead treated with a detergent (SDS) before the electrophoresis step. Thus, as concluded by the members of the IWGAV ${ }^{1}$, the results obtained for a given antibody in western blot applications cannot be used to predict the specificity of the antibody in another assay based on an entirely different epitope-retrieval method, such as IHC.

In the Human Protein Atlas (HPA) program, we have validated more than 24,000 in-house-generated antibodies directed to 17,000 human target proteins ${ }^{2}$. Although there is often a correlation between performance in different applications, we have observed many examples of antibodies that show strong support for specificity in IHC or immunofluorescence microscopy (IF) but which do not stain the correctly sized band in a western blot, and vice versa ${ }^{3-5}$. However, as pointed out by Lund-Johansen and Browning, western blot analysis has indeed been found to be useful as a general validation tool for antibody specificity. Many antibody providers use western blot analysis to show whether a band of the right size is stained or whether additional bands are present, the latter indicating off-target binding. This is indeed a practical procedure for a 'first-line' screening of antibodies for specificity. In the HPA program, we always screen our in-housegenerated antibodies by western blot analysis.

Similarly, protein arrays, in which the target protein is arrayed together with hundreds or thousands of unrelated proteins, are also useful tools for probing antibody specificity ${ }^{6}$. In this application, however, target proteins are often presented as purified (noncomplex) spots, and the proteins usually have not undergone any prior treatment with denaturing agents. This means that caution should also be applied when using protein arrays for validation of an antibody for its use in other applications, such as IHC and IF. 